\title{
Applications of electrostatic capacitance and charging
}

\author{
Titus Sandu, ${ }^{1}$ George Boldeiu, ${ }^{2}$ and Victor Moagar-Poladian ${ }^{2}$ \\ ${ }^{1}$ National Institute for Research and Development in Microtechnologies-IMT, \\ 126A, Erou Iancu Nicolae Street, 077190, Bucharest, ROMANIA \\ ${ }^{2}$ National Institute for Research and Development in Microtechnologies-IMT, \\ 126A, Erou Iancu Nicolae street, 07r190, Bucharest, ROMANIA
}

(Dated: January 9, 2014)

\begin{abstract}
The capacitance of an arbitrarily shaped object is calculated with the same second-kind integral equation method used for computing static and dynamic polarizabilities. The capacitance is simply the dielectric permittivity multiplied by the area of the object and divided by the squared norm of the Neumann-Poincaré operator eigenfunction corresponding to the largest eigenvalue. The norm of this eigenfunction varies slowly with shape thus enabling the definition of two scale-invariant shape factors and perturbative calculations of capacitance. The result is extended to a special class of capacitors in which the electrodes are the equipotential surfaces generated by the equilibrium charge on the object. This extention allows analytical expressions of capacitance for confocal spheroidal capacitors and finite cylinders. Moreover, a second order formula for thin constant-thickness capacitors is given with direct applications for capacitance of membranes in living cells and of supercapacitors. For axisymmetric geometries a fast and accurate numerical method is provided.
\end{abstract}

PACS numbers: 41.20.Cv, 82.47.Uv, 87.19.rf, 87.50.C-

\section{INTRODUCTION}

Potential theory has been proved very successfully in solving some boundary value problems such as the Dirichlet and the Neumann problems or the electrostatic charge distribution on conductors. For domains with sufficiently smooth boundaries (i.e., a regular piecewise Lyapunov surface) the above problems use specific types of potentials like the volume, the single-, and the double-layer potentials, the logarithmic potential for two-dimensional domains, etc. ${ }^{1,2}$. The Dirichlet and Neumann problems defined on domains with sufficiently smooth boundaries can be recast in integral equations which lead to compact operators on domain boundary: the NeumannPoincaré (or double-layer) operator and its adjoint ${ }^{2}$. These methods are applied in some practical and physical problems regarding dielectric heterogeneous systems like the radio-frequency and microwave dielectric spectra of living cells $\frac{3}{3}$ and plasmonic properties of metallic nanoparticles 4.5. Another problem is the equilibrium charge distribution on a conductor (the Robin problem) ${ }^{6}$ and the implicit capacitance with applications in computational biophysics ${ }^{7}$, in scanning probe microscopy ${ }^{8,9}$, or in electrical charge storage in supercapacitors ${ }^{10}$.

The capacitance of an arbitrarily shaped body is calculated by "mimicking" some directly related phenomena like the diffusion-controlled reactions ${ }^{11}$ or the ergodic generation of the equilibrium charge distribution ${ }^{12}$. The standard procedures for solving the Laplace equation are the Finite Element Method (FEM) ${ }^{13}$ or the Boundary Integral Equation (BIE) method with the finite element formulation as the Boundary Element Method (BEM) ${ }^{14}$. In contrast to the FEM, in the BEM only the surfaces of the inclusions are discretized, such that with numerical algorithms like the fast multipole method (FMM) of Rokhlin and Greengard ${ }^{15,16}$ the calculations are essen- tially of $O(N)$, where $N$ is the number of nodes. The capacitance of an arbitrary object has been treated in different contexts with $O(N)$ FMM schemes (see Ref. 17 and the references therein). It can be treated as a first-18 or as a second-kind integral equation. The second-kind integral formulation is based either on the NeumannPoincaré operator ${ }^{19}$ or on its adjoint 17 . The most convenient approach is, however, the second-kind integral equation with the adjoint of the Neumann-Poincaré operator which provides both the charge density and the capacitance 17 .

In this paper we adopt such a BIE method to calculate the capacitance and the equilibrium charge on an arbitrarily shaped object with several applications. The capacitance is obtained concurrently with other physical properties like the static and the dynamic polarizabilities of nanoparticles with applications in nanoparticle manipulation ${ }^{20}$ and plasmonics 5 . We use a spectral method ${ }^{21}-23$ which provides an exponential convergence 24 . Moreover, our basis functions include spherical harmonics ${ }^{21}$ that can be directly related with the multipoles in the FMM of Rokhlin 15,16. Compared to others the present method shows directly that the geometric dependence of capacitance is incorporated in a norm of a given eigenvector of the Neumann-Poincaré operator. This eigenvector norm varies slowly with the geometry hence two scale-invariant defined shape factors can be readily used in the estimation of capacitance for arbitrary shapes. Furthermore, we define a specific class of capacitors in which the electrodes are the equipotential surfaces generated by the equilibrium charge on an arbitrarily shaped metallic object with applications regarding some analytical results like confocal spheroidal capacitors and finite cylinders. We also provide a second order compact capacitance formula for thin and constantthickness capacitors with other applications referring to 
membrane capacitance of living cells and charge storage in supercapacitors.

The paper is organized as follows. In the second section we define the capacitance in the second-kind integral form. Then, we define a general capacitor and a specific class of capacitors in the following section. Section 4 describes the numerical method and the applications just mentioned above. A summary is given in the last section.

\section{CAPACITANCE OF A METALLIC OBJECT IN A SECOND-KIND INTEGRAL FORMULATION}

We assume an arbitrarily shaped domain $\Omega$ bounded by the surface $\Sigma$ in the 3 -dimensional space. The following operators can be defined on $\Sigma: \hat{M}$, its adjoint $\hat{M}^{\dagger}$, and $\hat{S}, \frac{1,2}{2}$ The action of $\hat{M}$ on a function $u$ signifies the normal electric field to $\Sigma$ generated by the charge density $u$. The operator $\hat{M}^{\dagger}$, which is the Neumann-Poincaré (or double-layer) operator, acts on the dipole density $v$ generating an electric potential on $\Sigma$. On the other hand, $\hat{S}$ is a Coulomb (single-layer) operator which acts on the charge density $u$ creating an electric potential on $\Sigma$.

The operators $\hat{M}$ and $\hat{M}^{\dagger}$ have the same spectrum within $-1 / 2$ and $1 / 2$ and the eigenfunctions $u_{i}$ of $\hat{M}$ are related to the eigenfunctions $v_{i}$ of $\hat{M}^{\dagger}$ by $v_{i}=\hat{S}\left[u_{i}\right]$, which makes them bi-orthogonal, i.e., $\left\langle v_{j} \mid u_{i}\right\rangle=\delta_{i j} 23$. The largest eigenvalue of $\hat{M}$ and $\hat{M}^{\dagger}$ is $1 / 2$ irrespective of the domain shape $e^{2,21}$ and the corresponding eigenfunction $v_{1}$ of $\hat{M}^{\dagger}$ is a constant function, i. e., $v_{1}=$ constant on $\Sigma$. As we will discuss below, the companion eigenfunction $u_{1}$ of $\hat{M}$ is proportional to the equilibrium charge distribution on a conductor of shape determined by $\Sigma$. We note that the spectrum of $\hat{M}$ and $\hat{M}^{\dagger}$ is scale invariant, but the spectrum of $\hat{S}$ is proportional to the linear size of $\Omega$.

$\hat{M}$ and $\hat{S}$ can be used in the resolution of many physical problems like the static $\underline{20}$ or the dynamic object polarizability represented by the dielectric spectra of living cells 3.21 or the optical properties of metallic nanoparticles $4,5,22,23$. Another closely related issue is the Robin problem of finding the equilibrium charge distribution $u_{R}$ on a conductor of arbitrary shape $\mathrm{e}^{6}$. It can be cast into an integral equation of the second-kind that has the operator form

$$
\hat{M}\left[u_{R}\right]=\frac{1}{2} u_{R}(\mathbf{x}),
$$

with the constraint $\int_{\mathbf{x} \in \Sigma} u_{R}(\mathbf{x}) d \Sigma(\mathbf{x})=1$. The constraint can be put in the following form $\left\langle 1 \mid u_{R}\right\rangle=1$, where 1 is the constant function of value 1 on $\Sigma$. Equation (11) has the obvious solution $u_{R} \propto u_{1}$. The constant value $V_{R}$ of the electric potential generated by $u_{R}$ is formally given by $\hat{S}\left[u_{R}\right]=V_{R} 1$ and is called the Robin constant, while its inverse is the capacitance $\mathrm{C}$ of the body bounded by $\Sigma$. If we consider the dielectric permittivity $\varepsilon$ of

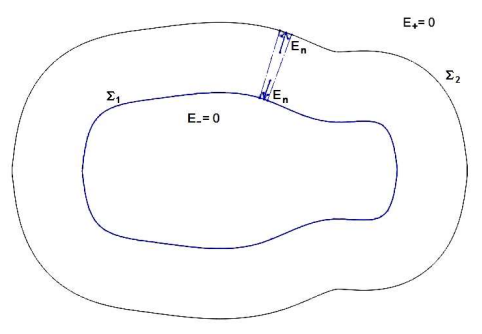

FIG. 1: Schematic representation of a capacitor with the configurations of fields inside $\Sigma_{1}$ and outside $\Sigma_{2}$. The dotted lines delimitate a Gaussian surface used in the text.

the embedding medium and the constraint $\left\langle 1 \mid u_{R}\right\rangle=1$ the capacitance is $C=\varepsilon /\left\langle u_{R} \mid \hat{S}\left[u_{R}\right]\right\rangle$. Furthermore, if $u_{R}=a_{1} u_{1}$, one can prove that the constant $a_{1}$ is the proportionality factor between $v_{1}$ and the constant distribution 1 , i. e., $1=v_{1} / a_{1}$. Then we can relate $a_{1}$ to the norm of $v_{1}$ by the following chain of equations $\left\langle v_{1} \mid v_{1}\right\rangle=\left\|v_{1}\right\|^{2}=a_{1}^{2}\langle 1 \mid 1\rangle=a_{1}^{2} A$, where $A$ is the area of $\Sigma$. Finally, the capacitance takes a simple and compact form

$$
C=\frac{\varepsilon A}{\left\|v_{1}\right\|^{2}} .
$$

One can show that $\left\|v_{1}\right\|^{2}$ is proportional to the linear size of $\Omega$ therefore, the capacitance itself is also proportional to the linear size of the body. Equation (2) shows explicitly both the geometric dependence of capacitance of an arbitrarily shaped object and the scale invariance of the shape factor $C / \sqrt{4 \pi A}$. The shape factor varies slowly with the conductor shape ${ }^{25}$ hence, as we will discuss in the next section, $\left\|v_{1}\right\|^{2}$ is a slowly varying function of the conductor shape and perturbative estimations of capacitance can be performed.

\section{CAPACITORS AND THEIR CAPACITANCE}

\section{A. Definition}

In general, a capacitor consists of two separated conducting bodies. We consider a capacitor that is made of two smooth surfaces $\Sigma_{1}$ and $\Sigma_{2}$ in which $\Sigma_{2}$ encloses $\Sigma_{1}$ (Fig. 11). The capacitance of the capacitor is defined as the total charge that is held on $\Sigma_{1}$ when the electrical potential is 1 on $\Sigma_{1}$ and 0 on $\Sigma_{2}$. Therefore, the electrostatic problem is the Laplace equation in the space $\Omega_{12}$ between $\Sigma_{1}$ and $\Sigma_{2}$ :

$$
\Delta u(\mathbf{x})=0 ; \mathbf{x} \in \Omega_{12}
$$

with the boundary conditions $u(\mathbf{x})=1$ for $\mathbf{x} \in \Sigma_{1}$ and $u(\mathbf{x})=0$ for $\mathbf{x} \in \Sigma_{2}$. It is easy to see that with these boundary conditions the solution of the Laplace equation inside of $\Sigma_{1}$ and outside of $\Sigma_{2}$ is the constant 1 and the 
constant 0 , respectively. Inside $\Omega_{12}$ we seek a solution for (3) in the form of two single-layer potentials

$$
u(x)=\int_{\mathbf{y} \in \Sigma_{1}} \frac{\mu_{1}(\mathbf{y})}{4 \pi|\mathbf{x}-\mathbf{y}|} d \Sigma(\mathbf{y})+\int_{\mathbf{y} \in \Sigma_{2}} \frac{\mu_{2}(\mathbf{y})}{4 \pi|\mathbf{x}-\mathbf{y}|} d \Sigma(\mathbf{y}),
$$

where $\mu_{1}$ and $\mu_{2}$ are the induced charge densities on $\Sigma_{1}$ and $\Sigma_{2}$. Similar to $\hat{M}$ and $\hat{S}$ we define on $\Sigma_{1}$ and $\Sigma_{2}$ four operators $\hat{M}_{i j}$ and four operators $\hat{S}_{i j}$ as follows

$$
\begin{gathered}
\hat{M}_{i j}\left[\mu_{j}\right]=\int_{\mathbf{x} \in \Sigma_{i} \mathbf{y} \in \Sigma_{j}} \frac{\mu_{j}(\mathbf{y}) \mathbf{n}(\mathbf{x}) \cdot(\mathbf{x}-\mathbf{y})}{4 \pi|\mathbf{x}-\mathbf{y}|^{3}} d \Sigma(\mathbf{y}), \\
\hat{S}_{i j}[u]=\int_{\mathbf{x} \in \Sigma_{i} \mathbf{y} \in \Sigma_{j}} \frac{u(\mathbf{y})}{4 \pi|\mathbf{x}-\mathbf{y}|} d \Sigma(\mathbf{y}),
\end{gathered}
$$

with $i, j=\overline{1,2}$. In Eq. (5) $\mathbf{n}$ is the normal vector to $\Sigma_{1,2}$. The equations obeyed by $\mu_{1}$ and $\mu_{2}$ are

$$
\begin{aligned}
& \hat{M}_{11}\left[\mu_{1}\right]+\hat{M}_{12}\left[\mu_{2}\right]=\frac{1}{2} \mu_{1} \\
& \hat{M}_{21}\left[\mu_{1}\right]+\hat{M}_{22}\left[\mu_{2}\right]=-\frac{1}{2} \mu_{2} \\
& \hat{S}_{11}\left[\mu_{1}\right]+\hat{S}_{12}\left[\mu_{2}\right]=1 \\
& \hat{S}_{21}\left[\mu_{1}\right]+\hat{S}_{22}\left[\mu_{2}\right]=0 .
\end{aligned}
$$

The first two equations of (77) set the normal fields on $\Sigma_{1}$ from inside and on $\Sigma_{2}$ from outside to zero, while the last two equations are the boundary conditions of (3). The solution of the first two equations in (7) is the solution of (3) up to multiplicative constants. The multiplicative constants are fixed by the last two equations of (7).

\section{B. A special class of capacitors}

The capacitance of the capacitor is the total charge on $\Sigma_{1}$ and depends on inter-surface operators $\hat{M}_{i j}$ and $\hat{S}_{i j}$. In the special case when $\Sigma_{2}$ is an equipotential surface determined by the equilibrium charge distributed on $\Sigma_{1}$ a compact capacitance formula can be deduced with the help of $\hat{M}$ and $\hat{S}$ only. It is not hard to see that solutions $\mu_{1}$ and $\mu_{2}$ of the first two equations of (7) are proportional to the equilibrium charge densities on $\Sigma_{1}$ and $\Sigma_{2}$, respectively. To determine $\mu_{1}$ and $\mu_{2}$ one needs the boundary conditions given by the last two equations of (77). Thus, by integrating the third equation of (7) on $\Sigma_{1}$ and the fourth equation on $\Sigma_{2}$ one obtains the following relations $V_{1}+V_{2}=1$ and $V_{12}+V_{2}=0$, where $V_{1}$ is the electric potential induced by $\mu_{1}$ on $\Sigma_{1}, V_{2}$ is the electric potential induced by $\mu_{2}$ inside $\Sigma_{2}$ as well as on $\Sigma_{1}$, and $V_{12}$ is the electric potential induced on $\Sigma_{2}$ by $\mu_{1}$. On the other hand, the total charges on $\Sigma_{1}$ and on $\Sigma_{2}$ are $Q_{1}=C_{1} V_{1}$ and $Q_{2}=C_{2} V_{2}$, which are valid only if $\Sigma_{2}$ is one of the equipotential surfaces determined by an equilibrium charge distributed on $\Sigma_{1}$. Equation (2) provides the expressions of $C_{1}$ and $C_{2}$ that are the capacitances of $\Sigma_{1}$ and $\Sigma_{2}$, respectively. Keeping in mind that $Q_{1}+Q_{2}=0$ we obtain the capacitance

$$
C_{\text {cond }}=\left(\frac{1}{C_{1}}-\frac{1}{C_{2}}\right)^{-1}
$$

In the limiting case of very thin capacitors (i. e., $\Sigma_{2}$ being very close to $\Sigma_{1}$ ) Eq. (8) takes a planar-like capacitor expression given by

$$
C_{\text {thin_capacitor }}=\varepsilon \int_{y \in \Sigma} \frac{d \Sigma(y)}{\delta d},
$$

where $\delta d$ is the "distance" between $\Sigma_{1}$ and $\Sigma_{2}$ locally defined below. In the vicinity of $\Sigma_{1}$ a coordinate system $\left(\zeta_{1}, \zeta_{2}, \zeta_{3}\right)$ can be defined, such that $\zeta_{1}$ and $\zeta_{2}$ describe $\Sigma_{1}$ while $\zeta_{3}$ is the electric potential following the field lines from $\Sigma_{1}$ to $\Sigma_{2}$. The electric potential $V_{2}$ on $\Sigma_{2}$ is related to $V_{1}$, the electric potential on $\Sigma_{1}$, by

$$
V_{2} \cong V_{1}+\frac{\partial V}{\partial \zeta_{3}} \delta \zeta_{3},
$$

where $\delta \zeta_{3}$ is a small variation of $\zeta_{3}$ from $\Sigma_{1}$ to $\Sigma_{2}$. The local thickness of the capacitor is $\delta d=h_{\zeta_{3}} \delta \zeta_{3}$, with $h_{\zeta_{3}}$ as the Lamé coefficient corresponding to $\zeta_{3} 26$. From Eq. (10) Gauss theorem (see for instance Fig. 11) provides the charge density $\sigma=\varepsilon\left(V_{1}-V_{2}\right) / \delta d$. Integrating the charge $\sigma$ over $\Sigma_{1}$ and dividing by $V_{1}-V_{2}$ one obtains (9). Now we consider without loss of generality that $\left(\zeta_{1}, \zeta_{2}, \zeta_{3}\right)$ is orthogonal. Then using Eq. (9) the form of Eq. (8) can be recast as

$$
C_{\text {cond }}=\varepsilon\left(\int_{1}^{0} \frac{d \zeta_{3}}{\int_{y \in \Sigma} \frac{d \Sigma(y)}{h_{\zeta_{3}}(y)}}\right)^{-1}
$$

The validity of (9) is more general than that of the case considered above (in which $\Sigma_{2}$ is an equipotential surface generated by the equilibrium charge on $\Sigma_{1}$ ). Some examples will be provided in the next section, where it will be also discussed cases in which (9) may not be good enough.

Particular examples of Eqs. (8) and (11) are the capacitances of concentric spheres and of coaxial cables. For a capacitor made of two concentric spheres the capacitance is $C_{\text {sph_cond }}=4 \pi \varepsilon R_{1} R_{2} /\left(R_{2}-R_{1}\right)$, where $R_{1}$ and $R_{2}$ are the radii of the two spheres with $R_{2}>R_{1}$. Since the capacitance of a sphere is $C_{s p h}=4 \pi \varepsilon R$, it is easy to check that $C_{s p h \_c o n d}$ has the form given by Eq. (8).

The capacitance of a capacitor made of two confocal spheroids obeys also (8) and can be calculated with Eq. (11). Two confocal spheroids are conveniently described in spheroidal coordinates $(\eta, \xi, \varphi)$, which for prolate spheroids obey the equations

$$
\begin{aligned}
& x=c \sqrt{\eta^{2}-1} \sqrt{1-\xi^{2}} \cos (\varphi) \\
& y=c \sqrt{\eta^{2}-1} \sqrt{1-\xi^{2}} \sin (\varphi) \\
& z=c \eta \xi
\end{aligned}
$$


The two confocal spheroids defining $\Sigma_{1}$ and $\Sigma_{2}$ are determined by $\eta=\eta_{1}$ and $\eta=\eta_{2}$, respectively. The coordinates $(\eta, \xi, \varphi)$ are orthogonal and it can be shown that the equipotential surfaces of the eqiulibrium charge on the spheroid of equation $\eta=\eta_{1}$ is any confocal spheroid of equation $\eta=\eta_{2}>\eta_{1}{ }^{26}$. From Eq. (11) one obtains directly the capacitance of a confocal spheroidal capacitor as in the following expression

$$
C_{\text {prolate_capacitor }}=\frac{4 \pi \varepsilon c}{Q_{0}^{0}\left(\eta_{1}\right)-Q_{0}^{0}\left(\eta_{2}\right)},
$$

where $Q_{0}^{0}(\eta)=\ln ((\eta+1) /(\eta-1))$. Eq. (13) is of form (8) since the capacitance of a prolate spheroid alone $\left(\eta_{2} \rightarrow \infty\right)$ is $C_{\text {prolate_spheroid }}=4 \pi \varepsilon c / Q_{0}^{0}\left(\eta_{1}\right)$ which is given in the standard textbooks of classical electrodynamics ${ }^{26}$. The capacitances of oblate confocal spheroids are found replacing $\eta$ by $i \eta$ and $c$ by $-i c$. An expression similar to (13) was found in a recent paper ${ }^{27}$, where the authors did not notice the significance of Eq. (13) in terms of Eq. (11).

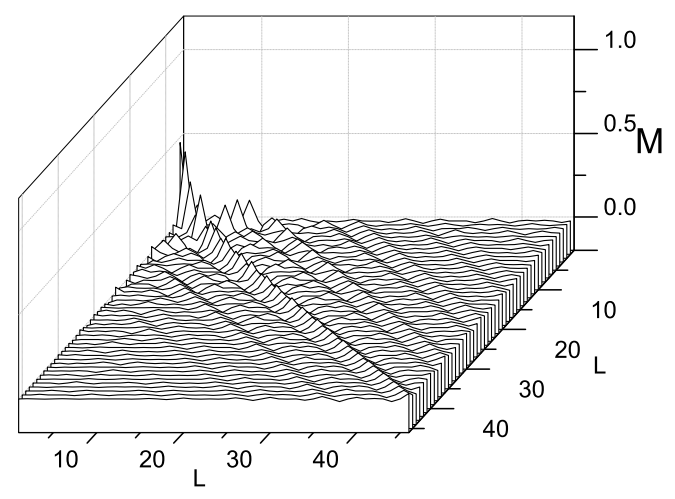

FIG. 2: Matrix elements of $\hat{M}$ in the $\tilde{Y}_{L m}$ basis. The matrix indices designate the indices $L$ of the spherical harmonics $\tilde{Y}_{L m}$. For axisymmetric objects only $m=0$ is relevant.

\section{APPLICATIONS AND DISCUSSION}

\section{A. A Numerical method}

The capacitance can be determined in numerical simulators used to calculate other physical properties like the dynamic polarizabilities needed for localized plasmon resonances in metallic nanoparticles 4,5 . Simultaneous calculations of capacitance and polarizability were also performed in the path integral formulation by averaging over random walk trajectories 28 . Our numerical method is an operator based BIE method that calculates the eigenvalues $\chi_{k}$ and the eigenvectors $u_{k}$ and $v_{k}$ of $\hat{M}$ and $\hat{M}^{\dagger}$, respectively. In order to have normed $u_{k}$ and $v_{k}$ one needs also to calculate the matrix elements of $\hat{S}^{23}$. The present method belongs to the class of the

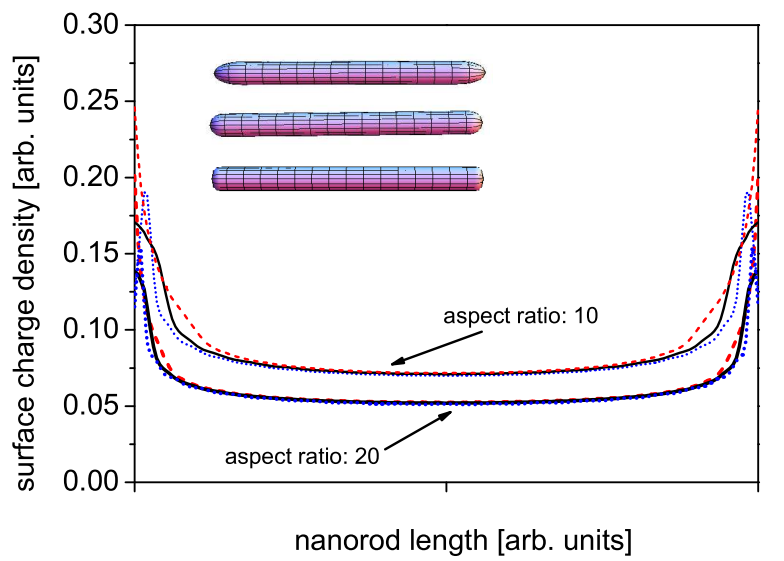

FIG. 3: (Color online) The equilibrium surface charge density on rods with various cappings shown in the inset: hemisphere (black solid line), oblate hemispheroid (blue dotted line), and prolate hemispheroid (red dashed line).

spectral methods which are fast converging 24 . In these methods the functions of the basis set are defined globally rather than locally like in the standard FEM. In our approach the function set is related to the spherical harmonics $Y_{L m}(\theta, \varphi)$ defined on a sphere that is related to $\Sigma$ by the map $\mathbf{x} \rightarrow(\theta(\mathbf{x}), \varphi(\mathbf{x}))$. Details of the method for axisymmetric objects are given in Refs. 21,22. In Fig. 2 we plotted the matrix elements of $\hat{M}$ for a generic axially symmetric object. It is easy to notice that the matrices are sparse with the significant matrix elements being around the diagonal or/and at low-value indices which are basically low-order multipoles. The matrix elements of $\hat{S}$ show also a similar behavior. Thus, our method is similar to the FMM in which the major contributions come from the low-order multipoles 15,16 . The validity of the numerical method was checked by calculations performed on oblate and prolate spheroids, which have analytical expressions discussed above. Our numerical calculations show a very good agreement with the analytical results. The relative error is at most $5 \times 10^{-5}$ with a relative small overhead of 25 functions in the basis and 96 quadrature points. The implementation of BIE for axisymmetric shapes has also shown to provide very accurate results of the depolarization factors which are related to other eigenvalues of $\hat{M}$ and $\hat{M}^{\dagger 29}$.

\section{B. Cylindrical rods}

We have also performed numerical calculations on cylindrical rods with different end-cap geometries: half of an oblate spheroid with $1 / 2$ aspect ratio, half of a sphere, and half of a prolate spheroid with an aspect ratio of 2. In Fig. 3 we have plotted the surface charge density of metallic rods with the above cappings. Two aspect ratios have been considered: 10 and 20. Since the charge preserves the axial symmetry only the lon- 
gitudinal dependence is shown. Fig. 3 illustrates that, ignoring the capping zones, the charge density is almost the same. The resemblance is greater as the aspect ratio becomes larger. In addition, in the middle of the rod the charge density is almost constant and decreases with the increase of the aspect ratio.

Let us now consider two hemispherically capped rods. The first one is determined by the surface $\Sigma_{1}$ that is a cylinder of length $L$ and caps of radius $R_{1}$. The other rod is determined by the outer surface $\Sigma_{2}$ with the same length $L$, but with a radius $R_{2}>R_{1} . \Sigma_{1}$ and $\Sigma_{2}$ have the same normal hence, geometrical intuition tells us that we can apply Eq. (11) to obtain the capacitance of a such capacitor made of two finite cylindrical rods with hemispherical ends. Explicit numerical calculations of equipotential surfaces show that the above assumption is quite good. Therefore, by applying Eq. (11) we obtain the following expression

$$
C_{\text {rod_capacitor }}=\frac{2 \pi \varepsilon L}{\ln \left(\frac{R_{2}\left(R_{1}+L / 2\right)}{R_{1}\left(R_{2}+L / 2\right)}\right)} .
$$

Eq. (14) recovers known results in two limiting cases: (a) concentric spheres, $L \rightarrow 0$; and (b) coaxial cable, $L \rightarrow \infty$. In the limiting case of $R_{2} \rightarrow \infty$ one obtains the capacitance of a finite cylindrical rod with hemispherical cappings

$$
C_{\text {hemispherical_rod }}=\frac{4 \pi \varepsilon R_{1}(m-1)}{\ln (m)},
$$

with $m=\left(L+2 R_{1}\right) /\left(2 R_{1}\right)$ as the aspect ratio of the rod.

This result can be extended to finite cylinders with hemispheroidal cappings where the equipotential surfaces are determined by the corresponding confocal spheroids. In the particular case of oblate hemispheroidal cappings one may obtain the limit of cylinder with flat cappings. Thus, after some tedious but otherwise straightforward calculations the capacity of cylinders with flat endings is

$$
C_{\text {flat_rod }}=\frac{4 \pi \varepsilon R\left(m^{2}+1\right)}{m\left(\ln (m)+\frac{\pi}{2 m}\right)},
$$

with $m=L /(2 R)$ as the aspect ratio of the rod. Here $L$ is the length of the rod and $R$ is its axial radius. We easily notice that for $m \rightarrow 0$ Eq. (16) reproduces the capacitance of a disk ${ }^{26}$.

We have compared numerical capacitance calculations of cylindrical rods having different capping geometries (flat, oblate hemispheroidal, and hemispherical) with Eqs. (15) and (16) and with Smythe's ${ }^{30}$ and Jackson's 31 analytical results. Numerical results are obtained using either the BIE method for rods with oblate hemispheroidal and hemispherical cappings or the multiphysics program ANSYS (found at wwww.ansys.com) for rods with flat cappings. The results are given in Fig. 4h. The oblate hemispheroidal cappings are chosen to be thin (an aspect ratio of 10), which provides quite good approximants for cylinders with flat ends. For example, the capacitances of flat and oblate hemispheroidal
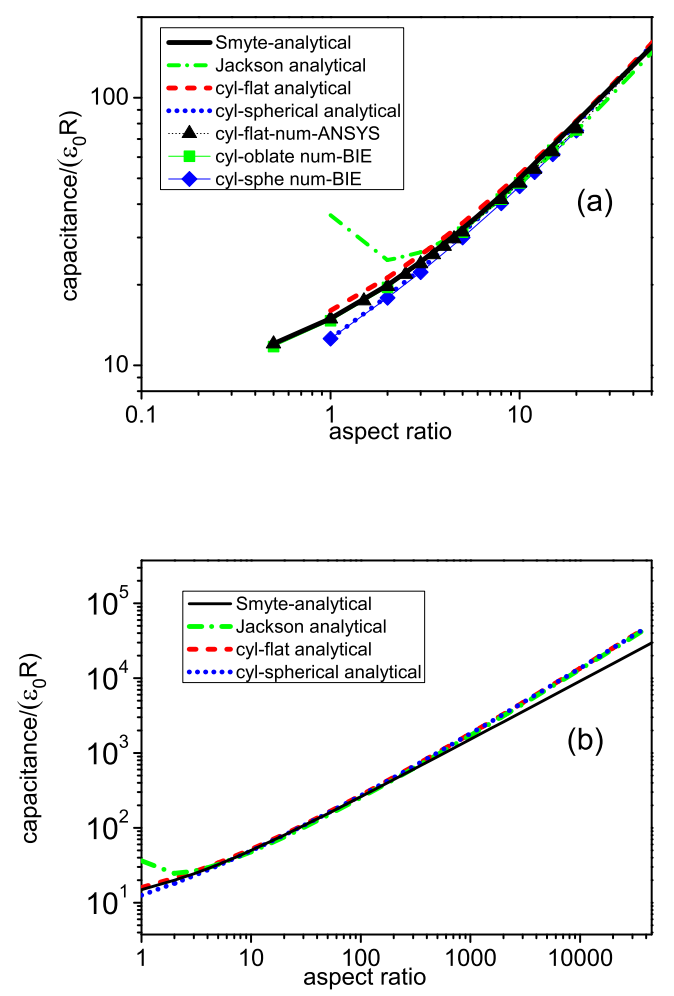

FIG. 4: (Color online) (a) Analytical and numerical calculations of capacitance for various cylindrical rods at relatively small aspect ratios. (b) Various analytical results over a sufficiently large range of aspect ratios. The capacitances are given in terms of $\varepsilon_{0} R$, with $\varepsilon_{0}$ as the vacuum permittivity and $R$ as the cross-sectional radius.

capped cylinders are apart only by $2 \%$ at an aspect ratio of $1 / 2$. Moreover, for aspect ratios greater than 5 the capacitance of the rods do not depend any longer on the end-cap geometry (the differences are well below 1\%). On the other hand, the analytical results of Eq. (15) are apart by up to $5.5 \%$ from the BIE calculations for cylinders with hemispherical ends at the aspect ratio of 20 . Furthermore, Eq. (16) is also within a few percentage points from the exact results at low aspect ratios, but at larger aspect ratios Eqs. (15) and (16) are sufficiently close. In Fig. 40 we compare Eqs. (15) and (16) with the Smythe's 30 and Jackson's 31 formulae. There are known that the Smythe's formula is valid at low aspect ratios (below 10$)^{30}$, while the Jackson's 31 works well at large aspect ratios. We mention that in another derivation there were obtained also two different expressions of capacitance for short and the long cylinders, respectively 32 . In contrast, as one can see from Fig. (4Eqs. (15) and (16) work well for both short and the long cylinders and also have simple algebraic expressions. These results are not that surprising after all. They are asymptotically exact for $m \rightarrow 0$ and $m \rightarrow \infty$ by construction. The case of very 
long cylinders was first considered by Maxwell ${ }^{33}$, who stated that, for $m \rightarrow \infty$, the charge density tends to be constant. As proved in a separate paper ${ }^{34}$ the charge and implicitly the capacitance given by Jackson ${ }^{31}$ are similar to those of Maxwell's (e.g., $C=4 \pi \varepsilon R m /(\ln (4 m)-1))$ which are accurate for large but not for small $\mathrm{m}^{\prime} s$.

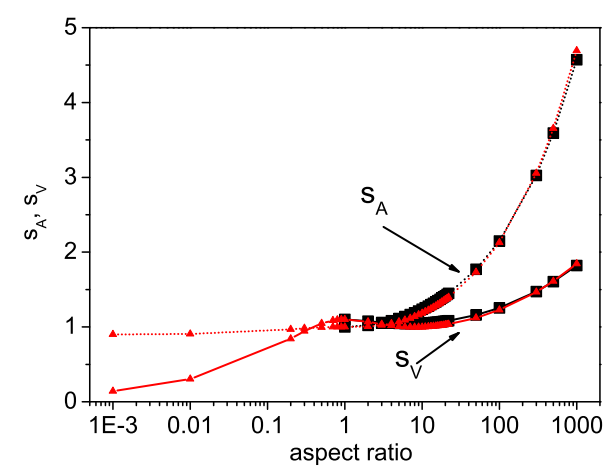

FIG. 5: (Color on-line) Scale-invariant shape factors versus aspect ratio. The shape factors are defined either by the area of the object ( $s_{A}$-dotted lines with symbols) or by its volume ( $s_{V}$-solid lines with symbols). The spheroids and the cylidrical rods are denoted by black squares and by red triangles, respectively.

\section{Scale-invariant shape factors and some consequences}

Since the capacitance is proportional to the linear size of the object one can define shape factors that are scaleinvariant. For instance one can employ the surface or the volume of the object to define scale-invariant shape factors. Two of such shape factors are presented in Fig. 5 for cylinders and spheroids.

The first scale-invariant shape factor defined as $s_{A}=$ $A^{1 / 2} /\left(2 \pi^{1 / 2}\left\|v_{1}\right\|^{2}\right)$ is related to the area $A$ of the object, such that it becomes 1 for spherical shape. It shows a relative shape insensitivity for aspect ratios less than 5 and for flat structures. This shape factor has been used in isoperimetric inequalities to estimates the capacitance of objects with shapes close to the spherical shape $e^{25}$.

The second scale-invariant shape factor related to capacitance is defined by $s_{V}=V^{1 / 3} /\left(\pi^{1 / 3}\left\|v_{1}\right\|^{2}\right)$. It is determined by the volume $V$ of the object and shows shape insensitivity for long structures (Fig. 51). Thus for aspect ratios from 5 to $40 s_{V}$ is almost 1 for both rods and spheroids. In contrast to $s_{A}, s_{V}$ varies not much for long structure, but it goes to 0 for flat structures.

These shape factors can be straightforwardly utilized in approximate capacitance calculations for metallic object of various shapes. For instance, the capacitance for smooth shape approximants of the object can be calculated with the BIE method and then we may amend the
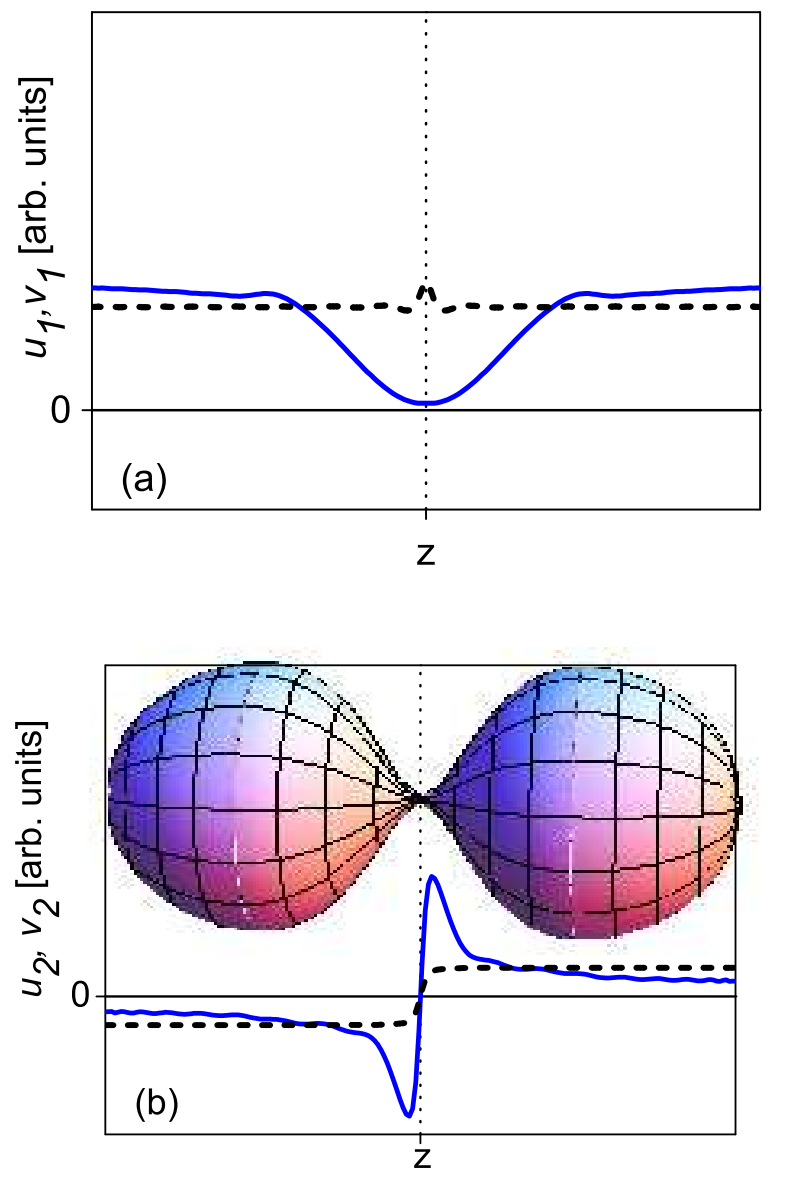

FIG. 6: (Color online) (a) The axial (z-) dependence of the first and (b) of the second eigefunctions of $\hat{M}$ and $\hat{M}^{\dagger}$ for a dimer; $u_{1}$ and $u_{2}$ are plotted with blue solid line, while $v_{1}$ and $v_{2}$ are depicted with black dashed lines. The inset of (b) shows the actual dimer.

final result with the appropriate area or volume by considering that the shape factor remains unchanged.

Our findings explain the $V^{1 / 3}$ scaling found for quantum capacitance of molecular nanowires 35 , at least for the aspect ratios $m$ from 5 to 30 . For long rods, on the other hand, the volume $V$ and the area $A$ of the rod scale almost linearly with $m$. But the quantum capacitance is in fact proportional to $\ln (m)$ (see Ref. 35), which turns out to be quite close to $m^{1 / 3}$ for $m$ between 5 and 30 . This simple remark explains the $V^{1 / 3}$ scaling of quantum capacitance in the same range of aspect ratios. So, for long structures the volume plays a greater role in determining both classical and quantum capacitances.

An interesting case is that of a dimer with slightly connected metallic particles of nearly spherical shape ${ }^{22,23}$ with the shape depicted in the inset of Fig. 6b. In these systems the area and the volume related shape factors should become equivalent or close to that since they scales as $R^{2}$ and $R^{3}$, respectively, $R$ being the radius of the particles (the particles in the dimer are not quite 
spheres but very close that). As a result the capacitance of the dimers scales with the radius of the constituent particles. Our numerical calculations show that both the area and the volume related shape factors are very close to 1 , i. e., $s_{A}=1.009$ and $s_{V}=0.986$. Now we consider the case of two just touching spheres like that treated in Refs. 36, 37, where analytical expressions of capacitance are provided. The capacitance of touching spheres is $36.37: C_{\text {dimer }}=(2 \ln 2) \times 4 \pi \varepsilon R \approx 1.386 \times 4 \pi \varepsilon R$. If we consider that $s_{A}=1$ we obtain a capacitance $C_{\text {dimer } A}=$ $2^{1 / 2} \times 4 \pi \varepsilon R \approx 1.4142 \times 4 \pi \varepsilon R$. Similarly if $s_{V}=1$ the capacitance is $C_{\text {dimer } V}=(8 / 3)^{1 / 3} \times 4 \pi \varepsilon R \approx 1.387 \times 4 \pi \varepsilon R$. It is easy to check that the two scale-invariant shape factors also reproduce with a good accuracy the results of the asymmetric dimers given in Ref. 36 .

In dimers made of slightly connected particles many eigenfunctions of $\hat{M}$ and $\hat{M}^{\dagger}$ are hybrid eigenfunctions of the constituent particles ${ }^{22,23}$. An example is provided in Figs. 6a and 6b, where the first two eigenfunctions of $\hat{M}$ and $\hat{M}^{\dagger}$ are plotted. The first eigenfunctions $u_{1}$ and $v_{1}$ of $\hat{M}$ and $\hat{M}^{\dagger}$, respectively are basically symmetric combinations of the first eigenfunctions in the constituent particles. At the same time, the second eigenfunctions $u_{2}$ and $v_{2}$ are antisymmetric combinations of the same first eigenfunctions of the constituent particles. We notice that the first eigenmode $u_{1}$ provides the charging while it can not be a plasmon mode in metallic nanoparticles 23 . In the space between the particles of the dimer the charge is repelled thus, the particles themselves repell each other (Fig. 63). The second eigenmode, however, is a plasmon active mode in the long wavelength range 22 . Since the shape of $v_{2}$ is also constant on each particle, the first and the second eigenmode of $\hat{M}$ lead to the reminiscence of $C(V, V)$ and $C(Q,-Q)$, respectively, when the particles of the dimer are separated ${ }^{36,38} . C(V, V)$ is the capacitance when the two particles are kept at the same potential $V$ and, at touching, turns into the dimer capacitance discused above. In contrast, $C(Q,-Q)$ is the capacitance when the two particles are charged with opposite charges $Q$ and $-Q$. It logarithmically diverges as the spheres approach the touching point ${ }^{36,38}$ since the charging mode transforms into a dipole-active mode. This behavior of $C(V, V)$ and $C(Q,-Q)$ is expected to hold for dimers of any shape as it has been recently found for ellipsoidal ${ }^{39}$ or other, more general ${ }^{40}$, shaped dimers. In addition, Fig. 6a is an illustative image of sphere repulsion when they are in $\operatorname{contact}^{37}$ and Fig. 6b provides a glimpse of sphere attraction when they are kept at a constant voltage difference ${ }^{37,38}$.

\section{Thin capacitors}

Another application is the estimation of capacitance in thin capacitors like the membrane capacitance in living cells. The shelled ellipsoidal model and the spheroidal model, in particular, are two of the most common models of living cells used in modeling the dielectric spectroscopy

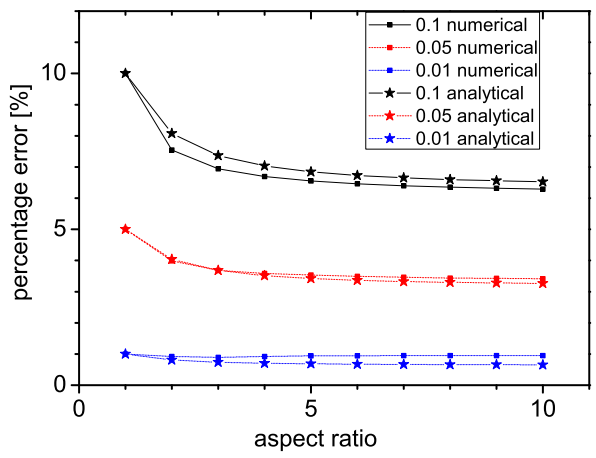

FIG. 7: (Color online) The percentage error of the planar-like capacitor formula with respect to the full numerical calculations (the square symbols) and with respect to analytical formula (the star symbols), i. e., Eq. (17), for thin constantthickness spheroidal capacitors.

experiments. In the spheroidal shelled model the shell designates the cell membrane, which is practically nonconductive and bounded by two confocal spheroids. Both the spherical ${ }^{41}$ and the spheroida ${ }^{42}$ models have analytical solutions for $\alpha$-(below the frequency of $10 \mathrm{KHz}$ ) and $\beta$ - (in the $\mathrm{MHz}$ range of the radiofrequency spectrum) relaxations 43 . On the other hand, the membrane capacitance needs to be estimated as an input parameter in the analysis of $\alpha$ - and $\beta$-relaxations ${ }^{41}$. Eq. (13) gives us also an analytical form of the membrane capacitance in the spheroidal model, putting the ellipsoidal and the spherical models on the same footing in terms of solvability. However, realistic cell models would imply constantthickness membrane, which is not found in the spheroidal model. In living cells, on the other hand, the membrane is very just a few tens of $n m$ thick for a cell size in the $\mu m$ range. Apparently, Eq. (9) is quite general for thin capacitors. The control parameter for accuracy is $\zeta_{1}$ which is weighted by its Lamé coefficient. Thus, it is of great interest to look for the validity of (9) in the case of thin and constant-thickness capacitors.

We suppose that $\Sigma_{1}$ is sufficiently smooth and let us pick an arbitrary point $\mathbf{r}_{0}$ on $\Sigma_{1}$ that is locally parametrized by $\mathbf{r}\left(\zeta_{1}, \zeta_{2}\right)$ and consider its two principal directions in which the curvature tensor is diagonal. We assume without loss of generality that $\zeta_{1}$ is the parameterization of the first principal direction and $\zeta_{2}$ the parameterization of the second. We further assume that the unit tangent vectors are $\mathbf{t}_{1,2}=\frac{\partial \mathbf{r}}{\partial \zeta_{1,2}}$, such that the surface element is $d \Sigma_{1}=d \zeta_{1} d \zeta_{2}$. Hence, the unit normal vector on $\Sigma_{1}$ at $\mathbf{r}_{0}$ is $\mathbf{n}=\mathbf{t}_{1} \times \mathbf{t}_{2}$. We also denote the curvature along the first principal direction at $\mathbf{r}_{0}$ as $\kappa_{1}$ and the curvature along the second principal direction at $\mathbf{r}_{0}$ as $\kappa_{2}$. In this situation the constant-thickness capacitor is determined by $\Sigma_{1}$ and by $\Sigma_{2}$ that is defined as $\mathbf{r}^{\prime}\left(\zeta_{1}, \zeta_{2}\right)=\mathbf{r}\left(\zeta_{1}, \zeta_{2}\right)+\delta a \mathbf{n}\left(\zeta_{1}, \zeta_{2}\right)$, where $\delta a$ is the is 
small and constant. Then the tangent vectors on $\Sigma_{2}$ are $\mathbf{t}_{1,2}^{\prime}=\mathbf{t}_{1,2}\left(1+\delta a \kappa_{1,2}\right)$. Therefore, in the linear approximation with respect to $\delta a$, the surface element on $\Sigma_{2}$ is $d \Sigma_{2}=d \zeta_{1} d \zeta_{2}(1+2 \delta a H)$, with the mean curvature $H=\left(\kappa_{1}+\kappa_{2}\right) / 2$. The key approximation that comes under scrutiny is Eq. (10), which is valid as long as the electric field on $\Sigma_{2}$ is the same as the electric field on $\Sigma_{1}$. According to the Gauss theorem the product of electric field and the surface element must be constant. In the linear approximation with respect to $\delta a$ the field $E_{2}$ on $\Sigma_{2}$ is $E_{2}=E_{1}(1-2 \delta a H)$, where $E_{1}$ is the field on $\Sigma_{1}$. Therefore, it is not hard to see that the voltage drop $V_{1}-V_{2}$ must be amended by the factor $(1-\delta a H)$ and Eq. (10) must be changed to

$$
C_{\text {membrane }}=\varepsilon \frac{A}{\delta a}+\int_{y \in \Sigma_{1}} \varepsilon H d \Sigma_{1}(y) .
$$

Eq. (17) is a second order approximation of thin constant-thickness, where the first term on the righthand side is the leading term. We have two straighforward consequences. First, Eq. (17) explains why the capacitance of cell membranes appear to be much larger whenever the membrane is folded ${ }^{44}$. Second, it gives us also the validity criterion of parallel-plane capacitor-like formula which is $\delta a \int H d \Sigma_{1}(y) / A \ll 1$. In Fig. 7 we present the percentage error of the planar-like capacitor formula, Eq.(9), with respect to full numerical calculations and with respect to Eq. (17) for thin constantthickness capacitors of prolate spheroidal shape. Three thicknesses are considered: $0.1,0.05$, and 0.01 of the largest axial cross-sectional radius of the spheroid. The calculations show that Eq. (17) is a good approximation of constant-thickness capacitors up to significant thichnesses. On the other hand, for extremely thin capacitors (a thickness of 0.01 ) the error of Eq.(9) is about $1 \%$.

A useful application of Eq. (17) is the assessment of the geometry-dependent energy storage in supercapacitors. In a recent paper Huang et al. ${ }^{45}$ noticed that the normalized capacitance (capacitance per unit area) of spherical and cylindrical double-layer capacitors increase with decreasing sphere and cylinder diameters. They also noticed that the capacitances of spherical capacitors increase faster than that of cylinders and argued that this behavior is related to the principal curvatures of those shapes 45 . Eq. (17) provides not only the proof for the curvature-related capacitance but also a quantitative evaluation of shape dependent capacitance. In addition, the same equation can be utilized for double-layer capacitors of arbitrary shapes.

\section{SUMMARY}

In the second-kind integral equation based on the adjoint of the Neumann-Poincaré operator the capacitance as well as the static and dynamic polarizabilities can be simultaneously calculated for arbitrarily shaped objects. A compact capacitance formula is obtained and is simply stated as follows. The capacitance is direct proportional to the dielectric permittivity of the embedding medium and to the area of the object, and inverse proportional to the squared norm of the eigenfunction of the NeumannPoincaré operator with the largest eigenvalue. A spectral based numerical implementation of the method is accurate and resembles the fast multipole method. Several applications are discussed. The capacitance formula allows us to define scale-invariant shape factors that varies slowly with shape and can be used in approximate calculations of capacitance. We have analyzed two scaleinvariant shape factors. One of the shape factors employs the volume of the object and is more suitable for long shapes like rods or wires. The other shape factor, which is defined in terms of the object area, is more appropriate for objects with shapes close to a sphere. Both scale-invariant shape factors, however, provide an accurate capacitance of touching metallic dimers.

We have extended the above results to capacitors. More explicitly, we have considered a special class of capacitors defined by the equipotential surfaces of the equilibrium charge on an arbitrarly shaped body. In this case the capacitor behaves like a series capacitor with the total capacitance as being the capacitance of the inner surface in series with the opposite (negative) capacitance of the outer surface of the capacitor. This result leads to an integral form of capacitance that was used to estimate analytically the capacitance of confocal spheroidal capacitors and of finite cylinders. Another consequence is a second-order formula for thin constant-thickness capacitors of arbitrary shape. The first order term has a planecapacitor like form, while the second order term is the surface integral of the mean curvature. Applications of a thin constant-thickness capacitor formula are encountered in the capacitance estimation of membrane in living cells and of supercapacitors with arbitrary shapes.

\section{Acknowledgments}

This work was supported by a grant of the Romanian National Authority for Scientific Research, CNCS - UEFISCDI, project number PNII-ID-PCCE-2011 -2-0069.
* Electronic address: titus.sandu@imt.ro

1 O. D. Kellog, Foundations of Potential Theory (SpringerVerlag, Berlin-Heidelberg-New York, 1967).

2 D. Khavinson, M. Putinar, and H. S. Shapiro, Arch. Ra- tion. Mech. Anal. 185, 143 (2007).

3 D. Vrinceanu and E. Gheorghiu, Bioelectrochem. Bioenerg. 40, 167 (1996).

${ }^{4}$ F. Ouyang and M. Isaacson, Philos. Mag. B 60, 481 (1989). 
${ }^{5}$ I. D. Mayergoyz, D. R. Fredkin, and Z. Zhang, Phys. Rev. B 72, 155412 (2005).

${ }^{6}$ G. Robin, Ann. Sci. Ecole Norm. Sup. 3, 1 (1886).

7 T. Simonson, Rep. Prog. Phys. 66, 737 (2003).

8 W. A. Hofer, A. S. Foster, and A. L. Shluger, Rev. Mod. Phys. 75, 1287 (2003).

9 A. Mottaghizadeh, P. L. Lang, L. M. Cui, J. Lesueur, J. Li, D. N. Zheng, V. Rebuttini, N. Pinna, A. Zimmers, and H. Aubin, Appl. Phys. Lett. 102, 053118 (2013).

10 P. Simon and Y. Gogotsi, Nature Mat. 7, 845 (2008).

11 J. F. Douglas, H. X. Zhou, and J. B. Hubbard, Phys. Rev. E 49, 5319 (1994).

12 M. Mascagnia and N. A. Simonov, J. Comput. Phys. 195, 465 (2004).

13 C. Johnson, Numerical Solutions of Partial Differential Equations by Finite Element Method (Cambridge Univ. Press, Cambridge, 1987).

14 D. Poljak and C. A. Brebbia, Boundary Element Methods for Electrical Engineers (WIT, Boston, 2005).

15 V. Rokhlin, J Comput. Phys. 60, 187 (1985).

16 L. F. Greengard and V. Rokhlin, J. Comput. Phys. 73, 325 (1987).

17 J. Tausch and J. White, Adv. Comput. Math. 9, 217 (1998).

18 K. Nabors, F. T. Korsmeyer, F. T. Leighton, and J. White, SIAM J. Sci. Statist. Comput. 15, 713 (1994).

19 A. Greenbaum, L. Greengard, and G. B. M. Fadden, J. Comput. Phys. 105, 267 (1993).

20 A. A. Farajian, O. V. Pupysheva, H. K. Schmidt, and B. I. Yakobson, Phys. Rev. B 77, 205432 (2008).

21 T. Sandu, D. Vrinceanu, and E. Gheorghiu, Phys. Rev. E 81, 021913 (2010).

${ }^{22}$ T. Sandu, D. Vrinceanu, and E. Gheorghiu, Plasmonics 6, 407 (2011).

23 T. Sandu, Plasmonics 8, 391 (2013).
24 J. P. Boyd, Chebyshev and Fourier Spectral Methods (Dover, New York, 2001).

25 Y. L. Chow and M. M. Yovanovich, J. Appl. Phys. 53, 8470 (1982).

${ }^{26}$ L. D. Landau and E. M. Lifshitz, Electrodynamics of Continuous Media (Pergamon, Oxford-New York, 1984).

27 O. D. Momoh, M. N. O. Sadiku, and C. M. Akujuobi, Microw. Opt. Tech. Lett. 51, 2361 (2009).

28 M. L. Mansfield, J. F. Douglas, and E. J. Garboczi, Phys. Rev. E 64, 061401 (2001).

29 T. Sandu, J. Nanopart. Res. 14, 905 (2012).

30 W. R. Smythe, J. Appl. Phys. 33, 2966 (1962).

31 J. D. Jackson, Am. J. Phys. 68, 789 (2000).

32 C. M. Butler, J. Appl. Phys. 51, 5607 (1980).

33 J. C. Maxwell, Proc. London Math. Soc. 9, 94 (1877).

34 J. D. Jackson, Am. J. Phys. 70, 409 (2002).

35 J. C. Ellenbogen, C. A. Picconatto, and J. S. Burnim, Phys. Rev. A 75, 042102 (2007).

36 J. Lekner, J. Electrostat. 69, 11 (2011).

37 J. Lekner, Proc. R. Soc. A 468, 2433 (2012).

38 J. Lekner, J. Appl. Phys. 111, 076102 (2012).

39 T. Murovec and C. Brosseau, Appl. Phys. Lett. 102, 084105 (2013).

40 A. S. Khair, J. Appl. Phys. 114, 134906 (2013).

41 E. Prodan, C. Prodan, , and J. H. Miller, Biophys. J. 95, 4174 (2008).

42 A. D. Biasio, L. Ambrosone, and C. Cametti, Biophys. J. 99, 163 (2010).

43 R. Stoy, K. Foster, and H. Schwan, Phys. Med. Biol. 27, 501 (1982).

44 C. M. Lo, C. R. Keese, and I. Giaever, Biophys. J. 69, 2800 (1995).

45 J. Huang, B. G. Sumpter, V. Meunier, G. Yushin, C. Portet, and Y. Gogotsi, J. Mater. Res. 25, 1525 (2010). 